\title{
Integrating the gastrointestinal sciences
}

\author{
Stephen J. Pandol* \\ University of California, Los Angeles, CA, USA \\ *Correspondence: stephen.pandol@va.gov
}

Our conventional approach to investigating the physiology and pathophysiology of the gastrointestinal tract most often focuses on specific pathways or functions of an organ often to the exclusion of the influences of other pathways, organs and even the intra-luminal contents of the gastrointestinal tract. This approach is strongly influenced by the need to provide hypothesis-driven research that includes controlling as many variables as possible in the experiment; and measuring specific responses to specific perturbations.

In recent years we are seeing a rapid expansion of exciting findings clearly demonstrating that long-term responses (both physiologic and pathophysiologic) are dependent on interplays between dietary constituents, gut microbes, gut epithelial and immune functions and systemic metabolism (Garrett et al., 2010). Disorders in one or more participants in this interplay and/or maladaptive responses to environmental influences facing the gastrointestinal tract are likely to underlie some of the most common and devastating medical problems of our time including the metabolic syndrome (obesity, diabetes mellitus, hypertension and liver and vascular diseases), inflammatory gastrointestinal diseases and predisposition to several cancers.

Two major discoveries in the past century created vastly new approaches and understanding of gastrointestinal function. Bayliss and Starling (1902) demonstrated that acid instilled into the duodenum caused exocrine pancreatic secretion despite severing the duodenal and pancreatic nerves. Further, they showed that an extract of duodenal mucosa injected into the animal mimicked the acid effect in the duodenum. These were remarkable discoveries because they demonstrated that the intestine can release substances into the blood that have effects on other organs challenging the prevailing concept of "nervism" espoused by Pavlov. Out of this finding grew the discipline of endocrinology and Bayliss' and Starling's secretin was the first hormone. The field has grown enormously as a result of their contribution and progressed to an understanding that there are also key interplays between hormones and the nervous system that are necessary for gastrointestinal regulatory functions.

Marshall (2005) and Warren (2005) received the Nobel Prize in Physiology and Medicine for their remarkable and unexpected discoveries that ulcer diseases in the stomach and duodenum were caused by the bacterium Helicobacter pylori. Their seminal observations challenged the prevailing hypotheses that disorders of gastric acid secretion were mainly responsible for the disease. Further, their findings have greatly spurred interest in gut microbiology and its role in physiology and pathophysiology. For example, investigators worldwide are now pursuing hypotheses relating the gut microbiota to the diseases and disorders listed above.

In addition to the importance of these seminal discoveries in creating new paradigms for approaches to gastrointestinalrelated functions, they fittingly show that the development and testing of interdisciplinary hypotheses can lead to our most profound scientific discoveries. Thus, even though significant challenges in deciphering the molecular pathways involved in cellular functions and disorders of the gastrointestinal organs remain, enhancing and testing hypotheses with considerations of the integrated responses that occur in the system and applying interdisciplinary approaches will lead to the next generation of key discoveries in the gastrointestinal sciences.

Frontiers in Gastrointestinal Sciences is committed to publishing and promoting sound scientific findings that lead this next generation of discoveries.

\section{REFERENCES}

Bayliss, W. M., and Starling, E. H. (1902). The mechanism of pancreatic secretion. J. Physiol. 28, 325-353.

Garrett, W. S., Gordon, J. I., and Glimcher, L. H. (2010).

Homeostasis and inflammation in the intestine. Cell $140,859-870$.

Marshall, B. (2005). Helicobacter connections. ChemMedChem 1, 782-783.

Warren, J. R. (2005). Helicobacter: the ease and difficulty of a new discovery (Nobel lecture). ChemMedChem 1, 672-685.

Received: 14 June 2010; accepted: 15 June 2010; published online: 07 September 2010.

Citation: Pandol SJ (2010) Integrating the gastrointestinal sciences. Front. Physio. 1:19. doi: 10.3389/ fphys.2010.00019

This article was submitted to Frontiers in Gastrointestinal Sciences, a specialty of Frontiers in Physiology.

Copyright (c) 2010 Pandol. This is an open-access article subject to an exclusive license agreement between the authors and the Frontiers Research Foundation, which permits unrestricted use, distribution, and reproduction in any medium, provided the original authors and source are credited. 